International Journal of Engineering \& Technology, $7(2.16)(2018) 84-87$
International Journal of Engineering \& Technology
WPC
Website: www.sciencepubco.com/index.php/IJET
Research Paper

\title{
Shelter Brazen Out Apprehension and Their Exposure in VANET
}

\author{
Dr. S. Ramani ${ }^{*}$, M.R. Senkumar², Manish Kumar ${ }^{3}$ \\ $1,2 \& 3$ Sreenidhi Institute of Science and Technology, Hyderabad. India \\ *Email:dr.ramani2017@gmail.com.
}

\begin{abstract}
Vehicular Ad hoc Networks (VANET) is an application of MANET, due to its high mobility node which became exceedingly challenging research area. Vehicles which are connected to the NET through OBU (On board unit) are treated as nodes normally with high mobility in VANET. These nodes are certainly have authentication to transceives the status and refuge signals through one control groove having a modest bandwidth. This may origin an increasing impinging to the channel especially in impenetrable traffic circumstance. In Vehicular Accident Prevention System, the refuge messages are transceives through OBU sporadically on the highway to all of their neighbors within hearing range. These refuge messages are time sensitive and have stringent delay requirements. For these kinds of serious issue, Broadcast Authentication Protocol and Safe Routing are proposed against DDoS Attacks. The DDoS attacks broadcast towards authentication protocol leads to foreclose enervated authenticator. VANET networks is strongly dependent on their security and time alone features through routing, which will be conferred in this paper. The proposed protocol aims to make periodic the refuge messages to the proper authenticated nodes, so as to reduce the load on control groove as well as to avoid the collision within the short area of the sender vehicle.
\end{abstract}

Keywords: VANET, Broadcast Authentication Protocol, DDoS, Safety messages, Vehicle.

\section{Introduction}

Due to the huge traffic, the death accidents also enormously increased even though extremely framed Highways. For the recent era of road traffic, designing an expeditious safety system on the road is a predominant and censorious concern. It leads the auspicious interest to have research activities in the area of VANET because of the miscellany services it can offer. These services fall into all kind of shelter applications and non safety applications. Geo location information can enrich travel experience with some of these services like safety, Internet access and weather forecast etc. People suffer highly wastage of time due to traffic congestion [1], so a demand has risen to develop an efficient refuge system. This paper is aim to propose a novel conception to have better communication between vehicles, and to prevent accidents and traffic collision. The vehicle being consider as node should have the complete hardware circuit enhance with radars, sensors and GPS modem etc.. to support the VANET. However it also has unique characteristics that make a distinction from other mobile ad hoc networks; the most significant characteristics are: selforganization, good mobility, road pattern restrictions, and distributed communication all these characteristics made VANETs environment a tricky for developing well-organized protocols. However the node's rapidly dynamic topology and high moving speed, there are still some challenges for the accomplishment of VANET. For instance, the vehicles in VANET may tend to disengage frequently from the network, neighbouring vehicles transform frequently and do not have inbuilt relationships among them. This circumstances leads to increases delay and high packet loss. So it is an important issue to design an well-organized protocol so as to conquer the problems influenced by mobility over VANET. There is no uncertainty is that the broadcast authentication is an efficient way to resolve the above said issue but due to high mobility of the nodes, tradition authentication strategies may not satisfy the excepted resolution. The broadcast authentication protocol against to DDoS attacks [2] to avoid debilitated authenticator. APSM (active precise significance model) is the improved broadcast authentication against DDoS attacks is tried.

\section{Related Work}

2.1. Broadcast Authentication Protocol Scheme Based on DBP-MSP and Safe Routing in WSN against Attacks: (Jiawei Chen, 2011) [2] Here broadcast authentication with message specific puzzle is introduced to avoid weak authenticators. The puzzle solving ability and method is being considered enfranchisement of authentication. But this approach consumes more energy, memory space and delay to resolve the puzzle.

2.2. Review Analysis on Vehicular Ad-Hoc Networks Security Issues and Challenges: (D.Sivabalaselvamani.et.al, 2016) [4] A various types of routing and security problems of VANET been analyzed and discussed. Security is the real zone under exchange to actualize the VANET. The investigation of assaults found that the aggressor goal is to assault the system layer straightforwardly or in a roundabout way, subsequently the directing convention must be ensure enough to keep the most sorts of assaults. Every arrangement must protect the security prerequisites like confirma- 
tion, trustworthiness, and security which are more overpowered. Vehicular Ad Hoc Networks is a developing and promising innovation, this innovation is a fruitful district for assailants, who will attempt to go up against the system with their vindictive assaults. This report gives an investigation about the present situation and answers for the improvement. Aside from guaranteeing accessibility of data that offers a protected driving conduct and a superior voyaging background, the system is a financial correspondence, and information administration empowering influence. In any case, despite the fact that advantages, data security dangers and protection issues represent a colossal test to VANET extension and utilization. A standout amongst the most fascinating parts of the system is the capacity of the system to self-sort out in an exceedingly portable system environment. This paper furnished with a concise representation of the system by portraying the system qualities, design, applications, correspondence examples, and security challenges.

\section{DDoS Attack}

DDoS stands Distributed Denial of Service attack. Through this attacker an attempt to prevent zombie user from the service in order to avoid huge traffic from multiple compromised systems. Since unsuspecting vehicle are treated as zombies to carry out the attacks it is difficult to trace down the actual attacker. If at all the attacker receives broadcast packets, it is very smithy to forward them, which leads to addition of energy consumption [3] for the receiver. The DDoS attacks become more efficient through creating wormholes between different parts of the network [7].

Validation: In Vehicular network the authentication can perfectly identified through validate the electronic signature of the vehicle owner, but which leads to high expensive on computational design hence it is liable to DDoS attacks.

\section{Basic Architecture}

The basic architecture of VANET depends upon three structures are Inter vehicles communication, vehicle to Roadside Unit (RSU) communication and Inter Roadside Unit communication. Unlike MANET in the VANET the vehicles are travel in fixed paths such as roads, highways and city roads etc. While designing the system architecture the VANET is consider as a part of MANETs. To enhance the perfect architecture design the vehicle should have a transceiver namely, on board unit (OBU) to transceives the signal through the three basic structures. The first structure is 'vehicle to vehicle', where transmitter and receiver are vehicles means the vehicles receive information from other vehicles in the network and distribute that information to other vehicles in the network.

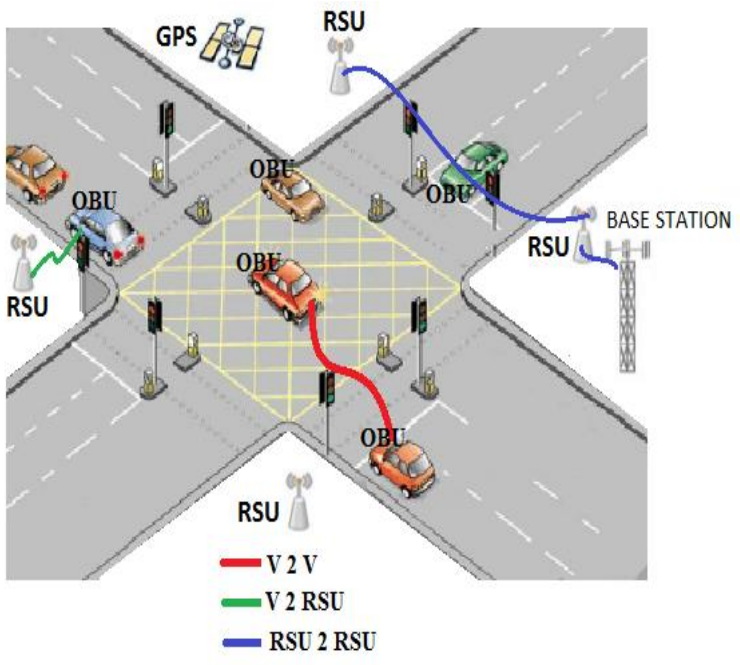

Figure.1 VANET Architecture
The second structure is 'vehicle to RSU' where either of transceiver is vehicle and infrastructure wireless component like RSU. In which RSU is used to collect information from vehicles and provide that information to other vehicles when necessary. The Hybrid structure is that a vehicle or RSU communicates with other nodes in single hop or multi hop.

VANETs are designed with the goals of enhancing driving safety and providing passenger comfort. The inter RSU Structure may look like wireless local area networks (WLAN). Figure 1 shows these three potential. VANET is mainly combination of an onboard unit (OBU) and more application units (AUs) [10]. An AU is a device implementing applications by using OBU's communication abilities. The both units of VANET are usually attached with a wireless or wired connection. The Ad-hoc domain incorporate the vehicles equipped with OBU and stationary units RSU sited along the road. The stationary road side and on board unit can be treated as nodes of vehicular ad-hoc network. RSU can be linked to an infrastructure network as well as to the Internet. RSU can also communicate to each other via multi-hop or directly. Their basic role is to enhancement of the road safety, by implementing special applications like sending, receiving or forwarding data in the ad-hoc domain.

\section{Energetic Precise Impact Pattern}

In the existing system, whenever the receiver receiving the packets it needs to solve the puzzle or carry through the electronic signature substantiation to forwarding the packets. Which leads to increase design complexity through the receiver execute the costly signature confirmation or do the packet transmission only when the solution is valid.

A novel Energetic Precise Pattern is pioneered along with safe routing. The creation of the authentication [9] is depends upon the design of the basic architecture and communication between the nature of three structures. A well known energy efficient hierarchical routing protocol called Base-Station Controlled Dynamic Clustering Protocol (BCDCP) [11] here is commenced to decrease the energy consumption. The receiver has to verify the implication method when an attacker forges a number of broadcast packets. Even though these fake packets may not be transceived through the receiver, when it comes to many numbers of times the higher appulse could be generated when aggregate natheless nodes are deployed in different locations and falsify a large number of broadcast packets.

\subsection{Corroboration}

\section{Handshake1: Sender node $\Rightarrow$ RSU: id}

The first message (Handshake) of the formation is nothing but the requisition for the authentication and every node should have its own unique identification ' $i d$ '. Once the base station receives the requisition, the status of the current broadcast workload could be updated to decide the difficult level called ' $N$ ' soon after it generates a random $N$-bit dynamic pattern 'P0'; it could be treated as a puzzle. In the intervening time, the respond value ' $\mathrm{n} 0$ ' is used as a factor in a hash function operation to produce a key ' $\mathrm{k} 0$ ', through this key the puzzle will be solved. Finally, the base station saves id, $\mathrm{n} 0, \mathrm{k} 0, \mathrm{P} 0$ in a broadcast state table.

\section{Memo 2: $R S U \Rightarrow$ Sender node: $\mathrm{PO}, \mathrm{nO}, \mathrm{kO}$}

The message 2 (memo 2) is nothing but the base station sends back the broadcast table which is preserved to the sender. Then sender will verify the solution and extend the solution with pattern $P 0$, key $k 0$, and respond value $n 0$ to solve the Energetic Precise Impact Pattern by brute-force testing. The suitable solution suppose to gratify the hash function, the first $\mathrm{k}$ bits in the image are $\mathrm{Pi}$ That is,

$$
\mathrm{H}(i|\mathrm{Mi} i n O| k 0|i d| \mathrm{Si})=\operatorname{Pixx} . . \mathrm{x}
$$

where "xx...x" can be any pattern, and Pi is the Energetic Precise Impact Pattern. In general the sender should analysis all practica- 
ble solution, to solve the puzzle by effective utilization of the Hash Function.

\section{Message i: Base station $\longrightarrow$ Sender node: $\mathrm{Pi}, \mathrm{ni}, \mathrm{ki}$}

The Final action of the current flow is nothing but, the base station sends a message $i$ to the sender with key ' $k i$ ' in every time interval ' $t$ ', and saves $P i, n i, k i$. With these values the sender solves the puzzle and forwards the packet with the solution $\mathrm{Si}$.

\subsection{Protected Steering Approach}

The next broad cast is between RSU and the receiver node. Before this the receiver examines and decides to perform the expensive digital signature verification through the help of base station.

\section{Handshake 1: Receiver node $\Rightarrow$ RSU:ni, ki, id}

In the handshake message the receiver sends the respond value ( $n i$ ) and key value $(\mathrm{ki})$ to the base station might be unique for a broadcast packet with a particular Energetic Precise Impact Pattern Pi. Also it is known that every node has its own unique id should also to update the broadcast state table to prevent replaying attacks.

\section{Memo 2: $R S U \Rightarrow$ Receiver node: $P i \mid N T$}

In this message first the RSU identifies the values $n i \& k i$ from the state table then broadcast the packets for the true values of ni, $k i \&$ $i d$. The base station also passes Pi to the sender. If not the true value, NT (Not true) will be set and then the packet will be dropped. Once the $\mathrm{Pi}$ is received then the receiver verifies the solution $\mathrm{Si}$ and come to a decision to perform expensive verifications.

\subsection{Revise the State Table}

The purpose of the state table is to identify false or replayed packets and to keep the difficulty level N. So it is mandatory to update the table and authentication strategy for every interval of time t. the receiver verify the $i d, n i, k i, P i$ through base station and for every Pi the $k i \& n i$ should match only then Pi will passed to the receiver. If not the RSU sets NT and drops the packet. Once all the packets are broadcasted to the entire node then the records in the state table will be cleared and receiver may not have Pi the network will be reset.

\section{Algorithm: To Revise the State Table}

Let 'P' denotes packets that RSU or (base station) receiving which serves as a request to broadcast made by the sender or for verification made by the receiver. 'Record' denotes the broad state table with records $\langle$ ni, ki $\rangle$ as index, $\langle\mathrm{Pi}\rangle$ as Energetic Precise Pattern, $<$ id $>$ as the records of nodes having forwarded the packet, and $<$ respond > as the number of them. $\mathrm{n}$ denotes the number of packets broadcasting in the network

Step 1 : Check for the 'id' is valid or not to send the packets $P$

Step 2 : Update the Record (State table) for every $(n+1)$ with the current values of ni, ki, id, Pi

$$
\text { So } \mathrm{n}=\mathrm{n}+1
$$

Repeat the step for the values 1 to $n$

Step 3 : Set the respond value depends on the number of packets

Step 4 : Check the new valid 'id' if it is false go to the step 7 or step 5.

Step 5 : Set the new indexed value ni, ki for the $\mathrm{P}$

Step 6 : Till the respond values clears update all the indexed value for new id.

\section{Step 7 : Set id is NT \\ Step 8 : Repeat all valid packets to forward. \\ Step 9 : RESET}

\section{Safety Investigation}

The authentication primarily depends on the generated key chain by sender through hash function [9]. More over almost all the cases it is the responsibility of the sender to generate the key chain. So a powerful sender i.e with high memory and computational capacity is required for an efficient network. At high traffic and the seed key $\mathrm{k} 0$ is been wrapped then the authentication become weakened [3]. Once the key is stolen by using the key $\mathrm{k} 0$ it is possible that an attacker to falsify many number of packets and attack other nodes in the network. To overcome this issue the generated key chain can be created for all senders were this could not viable by attacker. The revelation of one of the chains will not help an attacker to compromise another one. This also leads to reduce the required memory consumption significantly and the computation complexity also reduced dramatically which naturally wider the scope of application. Many numbers of trails is expected to solve the puzzle through hash function an attacker cannot have any kind of pre computed answer due to the $\mathrm{Pi}$, and hence cannot provoke competent DDoS attacks.

\section{Conclusion}

An original authentication method based on Energetic Precise Impact Pattern and safe routing strategy in VANET is proposed. Through powerful sender and mitigate DDoS attacks the proposed method overcome the limitation of the basic message passing significance scheme. Through the generated key chain method the energy and memory consumption of the sender is reduced. With nice property against DDoS attacks when the broadcast workload is heavy the application in VANET has became broader and strengthened.

\section{References}

[1] Anshula Malik, Jasbeer Narwal, 'Stability Improvement in GPSR in VANET' International Journal of Advanced Computing Research Volume 02- Issue 01, Jul 2016.

[2] [2].Jiawei Chen 'Broadcast Authentication Protocol Scheme Based on DBP-MSP and Safe Routing in WSN against DDoS Attacks' 2011 2nd International Conference on Networking and Distributed Computing, 978-0-7695-4427-4/11 2011 IEEE DOI 10.1109/ICNDC.2011.41

[3] [3].Ghassan Samara, Tareq Alhmiedat, Amer O. Abu Salem, 'Dynamic Safety Message Power Control in VANET Using PSO'World of Computer Science and Information Technology Journal (WCSIT) ISSN: 2221-0741 Vol. 3, No. 10, 176-184, 2013

[4] [4].Y. Sankarasubramaniam, I.F. Akyildiz, W. Su, E. Cayirci, "Wireless Sensor Networks: A Survey," Computer Networks 38 (2002) 393- 422.

[5] [5]. Perrig, R. Szewczyk, V. Wen, D. Culler, J. D. Tygar, "SPINS: Security Protocols for Sensor Networks," Mobile Computing and Networking 2001 Rome, Italy.

[6] [6]. N. Gura, A. Patel, A. Wander, H. Eberle, S. C. Shantz, "Comparing elliptic curve cryptography and RSA on 8-bit CPUs," In Proceedings of the 11th Network and Distributed Systems Security Symposium (NDSS '04). 17-36.

[7] [7]. P.Ning, A. Liu W. Du, "Mitigating DoS Attack against Broadcast Authentication in Wireless Sensor Networks," ACM Journal Vol., No., 20, Pages 1-31.

[8] [8].D. Liu, P. Ning, "Efficient Distribution of Key Chain Commitments for Broadcast Authentication in Distributed Sensor Networks" Washington DC:ACM Press, 2003:52-61.

[9] [9].D. Liu, P. Ning, "Multi-level uTESLA: Broadcast Authentication for Distributed Sensor Networks," ACM Transactions in Embedded Computing Systems 3, 4, 800-836. 
[10] [10].L. Hu, D. Evans, "Using Directional Antennas to Prevent Wormhole Attacks," In Proceeding of the 11 th Network and Distributed System Security Symposium, February 2003, pp. 131-141.

[11] [11].Siva D. Muruganathan, Daniel C. F. Ma, Rolly I. Bhasin, Abraham O. Fapojuwo, "A Centrialized Energy-Efficient Routing Protocol for Wireless Sensor Networks," IEEE Radio Communications March 2005 S8-S13.

[12] [12].X. Du, M. Guizani, Y. Xiao, H. Chen, "Defending Dos Attack on Broadcast Authentication in Wireless Sensor Networks," In Proceeding of IEEE Communication Society subject matter experts for publication in the ICC 2008. 